\title{
Spectroscopic signatures of spin-charge separation in the quasi-one-dimensional organic conductor TTF-TCNQ
}

Claessen, R.; Sing, M.; Schwingenschlogl, U.; Blaha, P.; Dressel, M.; Jacobsen, Claus Schelde

Published in:

Physical Review Letters

Link to article, DOI:

10.1103/PhysRevLett.88.096402

Publication date:

2002

Document Version

Publisher's PDF, also known as Version of record

Link back to DTU Orbit

Citation (APA):

Claessen, R., Sing, M., Schwingenschlogl, U., Blaha, P., Dressel, M., \& Jacobsen, C. S. (2002). Spectroscopic signatures of spin-charge separation in the quasi-one-dimensional organic conductor TTF-TCNQ. Physical Review Letters, 88(9), 096402. https://doi.org/10.1103/PhysRevLett.88.096402

\section{General rights}

Copyright and moral rights for the publications made accessible in the public portal are retained by the authors and/or other copyright owners and it is a condition of accessing publications that users recognise and abide by the legal requirements associated with these rights.

- Users may download and print one copy of any publication from the public portal for the purpose of private study or research.

- You may not further distribute the material or use it for any profit-making activity or commercial gain

- You may freely distribute the URL identifying the publication in the public portal 


\title{
Spectroscopic Signatures of Spin-Charge Separation in the Quasi-One-Dimensional Organic Conductor TTF-TCNQ
}

\author{
R. Claessen, ${ }^{1}$ M. Sing, ${ }^{1}$ U. Schwingenschlögl, ${ }^{1}$ P. Blaha, ${ }^{2}$ M. Dressel, ${ }^{3}$ and C. S. Jacobsen ${ }^{4}$ \\ ${ }^{1}$ Experimentalphysik II, Universität Augsburg, D-86135 Augsburg, Germany \\ ${ }^{2}$ Institut für Physikalische und Theoretische Chemie, Technische Universität Wien, A-1060 Wien, Austria \\ ${ }^{3}$ 1. Physikalisches Institut, Universität Stuttgart, D-70550 Stuttgart, Germany \\ ${ }^{4}$ Department of Physics, Technical University of Denmark, DK-2800 Lyngby, Denmark
}

(Received 1 August 2001; published 13 February 2002)

\begin{abstract}
The electronic structure of the quasi-one-dimensional organic conductor TTF-TCNQ is studied by angle-resolved photoelectron spectroscopy (ARPES). The experimental spectra reveal significant discrepancies to band theory. We demonstrate that the measured dispersions can be consistently mapped onto the one-dimensional Hubbard model at finite doping. This interpretation is further supported by a remarkable transfer of spectral weight as a function of temperature. The ARPES data thus show spectroscopic signatures of spin-charge separation on an energy scale of the conduction bandwidth.
\end{abstract}

DOI: 10.1103/PhysRevLett.88.096402

PACS numbers: 71.20.-b, 71.10.-w, 79.60.-i

The Fermi liquid (FL) concept is a central paradigm of solid state physics. It describes the low-lying electronic excitations of metals in terms of renormalized and only weakly interacting quasiparticles. Recently, various materials have been discovered whose unusual low-energy properties signal a breakdown of the quasiparticle picture. The theoretically best established case of non-FL physics is that of the interacting one-dimensional (1D) electron gas [1]. Here the quasiparticle picture fails due to a decoupling of charge and spin degrees of freedom, leading to the emergence of a new generic many-body quantum state commonly referred to as Luttinger liquid. Precise results have been obtained for its spectral properties at very low excitation energies [1,2]. Recently, several theoretical studies [3-7] have pointed out that in the case of strong local Coulomb interaction signatures of spin-charge separation may also be observable at high energies on the scale of the conduction bandwidth.

Experimentally, the search for Luttinger liquid signatures in quasi-1D metals has so far been directed at their low-energy spectral behavior. For example, virtually all quasi-1D metals studied by angle-resolved photoelectron spectroscopy (ARPES) display a characteristic suppression of spectral weight near the Fermi level $[8,9]$. However, this finding alone is not sufficient as manifestation of generic non-FL physics. Therefore, an unambiguous and direct spectroscopic identification of spin-charge separation in a 1D metal is still lacking.

In this Letter we present ARPES results on the electronic structure of the quasi-1D organic conductor TTF-TCNQ (tetrathiafulvalene tetracyanoquinodimethane) which reveal significant discrepancies from a conventional band structure description. We demonstrate that the TCNQrelated part of the experimental spectra can be consistently mapped onto the 1D Hubbard model at finite doping and thus reflects signatures of spin-charge decoupling. This interpretation is further supported by the observation of a temperature-dependent transfer of spectral weight over an energy scale of the bandwidth.

TTF-TCNQ is an organic charge transfer salt whose anisotropic properties can be understood from its monoclinic crystal structure [10,11], depicted in Fig. 1. Planar TTF and TCNQ molecules form separate linear stacks along the crystallographic b direction. Molecular orbitals of $\pi$ symmetry overlap with those of neighboring molecules stacked above and below. The covalent bonding along $\mathbf{b}$ is maximized by a rotation of the molecular planes about the a axis. A charge transfer of $\sim 0.59$
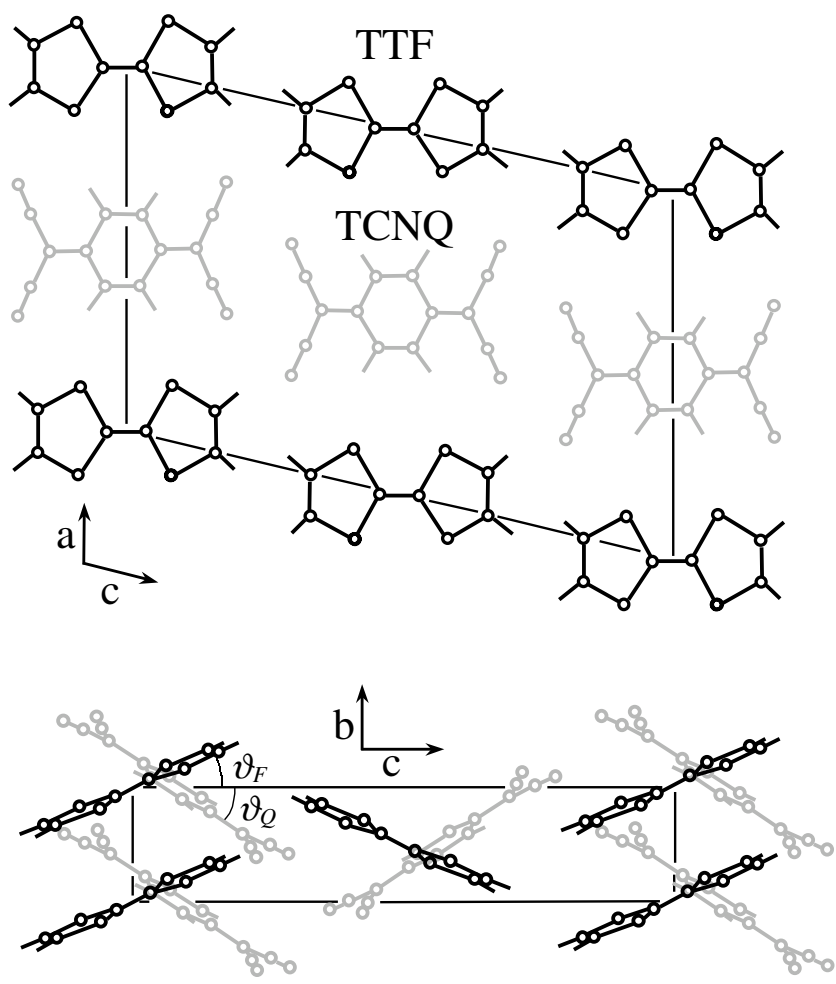

FIG. 1. Crystal structure of TTF-TCNQ. 
electrons per molecule from TTF to TCNQ drives both types of stacks metallic. The conductivity along $\mathbf{b}$ is up to 3 orders of magnitude larger than perpendicular to it, making TTF-TCNQ a truly quasi-1D metal. This is also reflected by a Peierls instability occurring at $T_{P}=54 \mathrm{~K}$.

There is substantial experimental evidence that Coulomb interaction plays an essential role in the electronic structure of TTF-TCNQ [11,12]. The effect of strong electronic correlations can approximately be described by the single-band 1D Hubbard model, with the on-site (i.e., intramolecular) Coulomb energy $U$ and the hopping integral $t$ as parameters. Various properties of TTF-TCNQ have successfully been analyzed within this approach [11]. There is general agreement that for TTF-TCNQ the ratio $U / 4 t \sim 1$, with the unperturbed bandwidth $4 t \sim 0.5 \mathrm{eV}$ from experiment and band theory $[11,12]$.

ARPES essentially measures the electron removal part of the spectral function $A(k, \omega)$ [13]. The experiments were partly performed at the U2/FSGM undulator beam line of BESSY I in Berlin, using an Omicron AR 65 spectrometer [14]. Spectra on the temperature dependence were taken at our home lab using $\mathrm{He}$ I radiation from a rare gas discharge lamp. In both cases the energy and momentum resolution amounted to $60 \mathrm{meV}$ and $0.07 \AA^{-1}$, respectively. All data were taken above the Peierls transition. Clean surfaces parallel to the ab plane were obtained by in situ cleavage of the crystals at a base pressure of $<10^{-10}$ mbar. Surface quality was checked by $\mathrm{x}$-ray photoemission [15]. All data presented here were measured before notable radiation-induced surface damage occurred.

The ARPES spectra measured along the $\mathbf{b}$ axis are shown in Fig. 2 and display pronounced dispersions. Their existence and periodic symmetry indicate a long-range surface order consistent with the bulk periodicity along $\mathbf{b}$. Spectra measured perpendicular to $\mathbf{b}$ are dispersionless (not shown here), as expected for a 1D metal. Our data are in excellent agreement with those of Zwick et al. [16] but partly display more spectral detail.

We now turn to a detailed discussion of the dispersions in Fig. 2. At zero momentum $(k=0)$ we can clearly identify two peaks at 0.19 and $0.54 \mathrm{eV}$ below $E_{F}$. With increasing wave vector the intensity dip between them gets smeared out leading to a broad intensity distribution between 0.15 and $0.6 \mathrm{eV}$, until at $k=0.16 \AA^{-1}$ again two peaks are resolved. This peculiar behavior is consistent with a splitting of the $0.54 \mathrm{eV}$ peak at $k=0$ into two separate structures, one moving to higher binding energy (labeled $d$ in Fig. 2) and one (b) dispersing towards the Fermi level, until it eventually merges with the low binding energy peak $a$ leading to the intense structure near the Fermi level at $k \approx 0.16, \ldots, 0.24 \AA^{-1}$. For even higher momenta a weak structure $c$ moves back again from the Fermi level and displays a dispersion symmetric about $k=0.87 \AA^{-1}$, corresponding to the Brillouin zone edge ( $Z$ point [17]). Simultaneously, structure $d$ disperses to higher binding energy and eventually becomes obscured by peak $c$. For

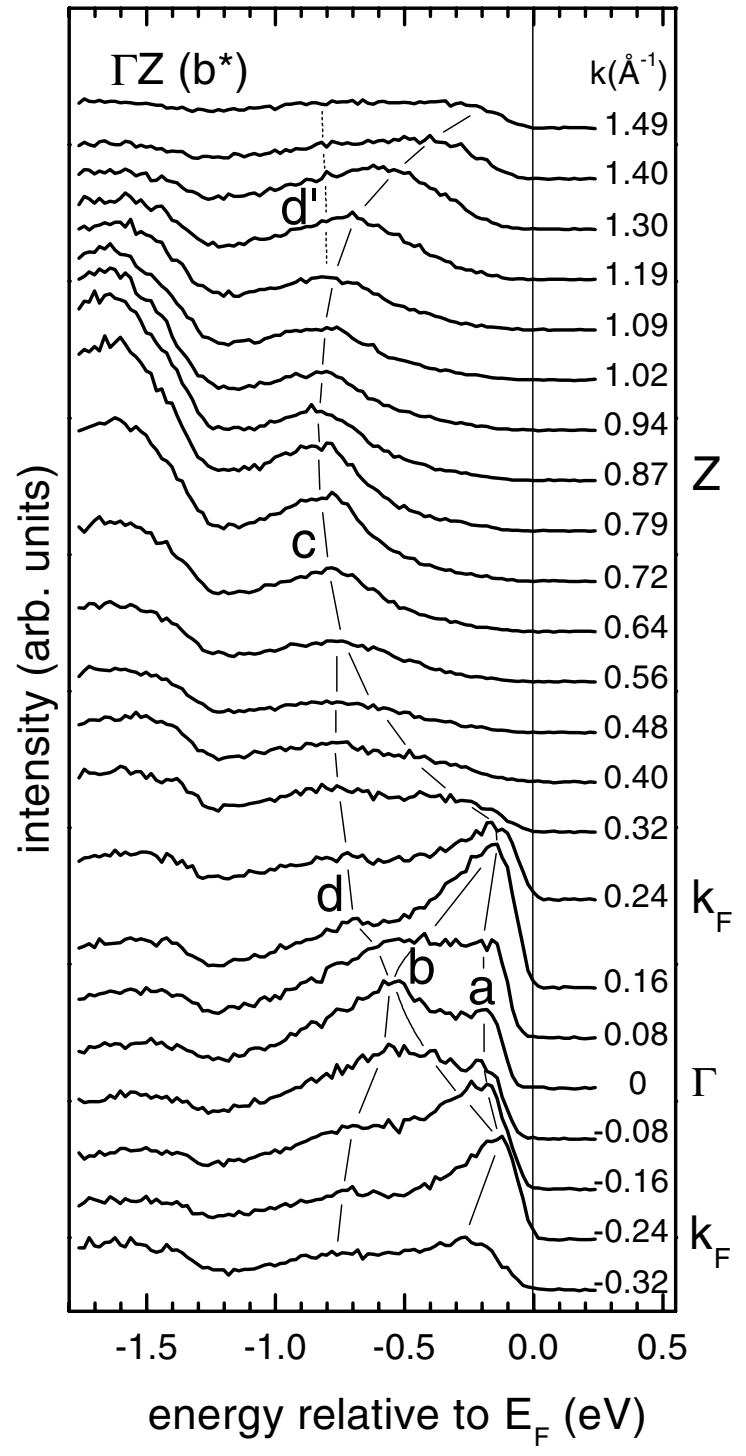

FIG. 2. Angle-resolved photoemission spectra measured for wave vectors $k$ along the $\Gamma Z$ direction $(h \nu=$ $25 \mathrm{eV}, T=61 \mathrm{~K})$. The thin lines indicate the dispersion of the spectral features.

wave vectors in the next zone a symmetry-related weak shoulder $d^{\prime}$ is observed.

Close to $k=0.24 \AA^{-1}$ spectral features $a, b$, and $c$ reach their closest approach to the Fermi level. We identify this position as the Fermi vector, even though no actual Fermi edge is observed, as already noted by Zwick et al. [16]. Rather, the intensity is suppressed almost linearly right down to the Fermi energy. We thus obtain a Fermi surface nesting vector of $2 k_{F}=0.48 \pm 0.06 \AA^{-1}$, in good agreement with the modulation vector of the Peierls state [11].

Figure 3(a) displays the ARPES spectra as a gray-scale plot of their negative second energy derivative in the $(E, k)$ plane, which enhances the visibility of the spectral structures and their dispersion. Also shown is our density functional band calculation [18], which yields two pairs of nearly degenerate bands attributed to the TCNQ chains and 
TTF chains, respectively. The qualitative behavior of experimental structures $a, b$, and $c$ is found to be in good correspondence with band theory. Peaks $a$ and $b$ are thus attributed to the TCNQ chains, while $c$ is assigned to the TTF stacks. Quantitatively we find, however, that the experimental bandwidths exceed those of the calculation by a factor of $\sim 2$. The fact that band theory is otherwise in good agreement with bulk properties indicates that at the surface, i.e., in the topmost molecular layers probed by ARPES, the hopping integral $t$ and therefore the bandwidth are renormalized. One may speculate that this effect is caused by a molecular surface relaxation; it is, however, of little relevance for the following discussion.

A much more serious problem of band theory is its failure to account for the experimental feature $d$. An interpretation in terms of a surface state seems tempting, but is in
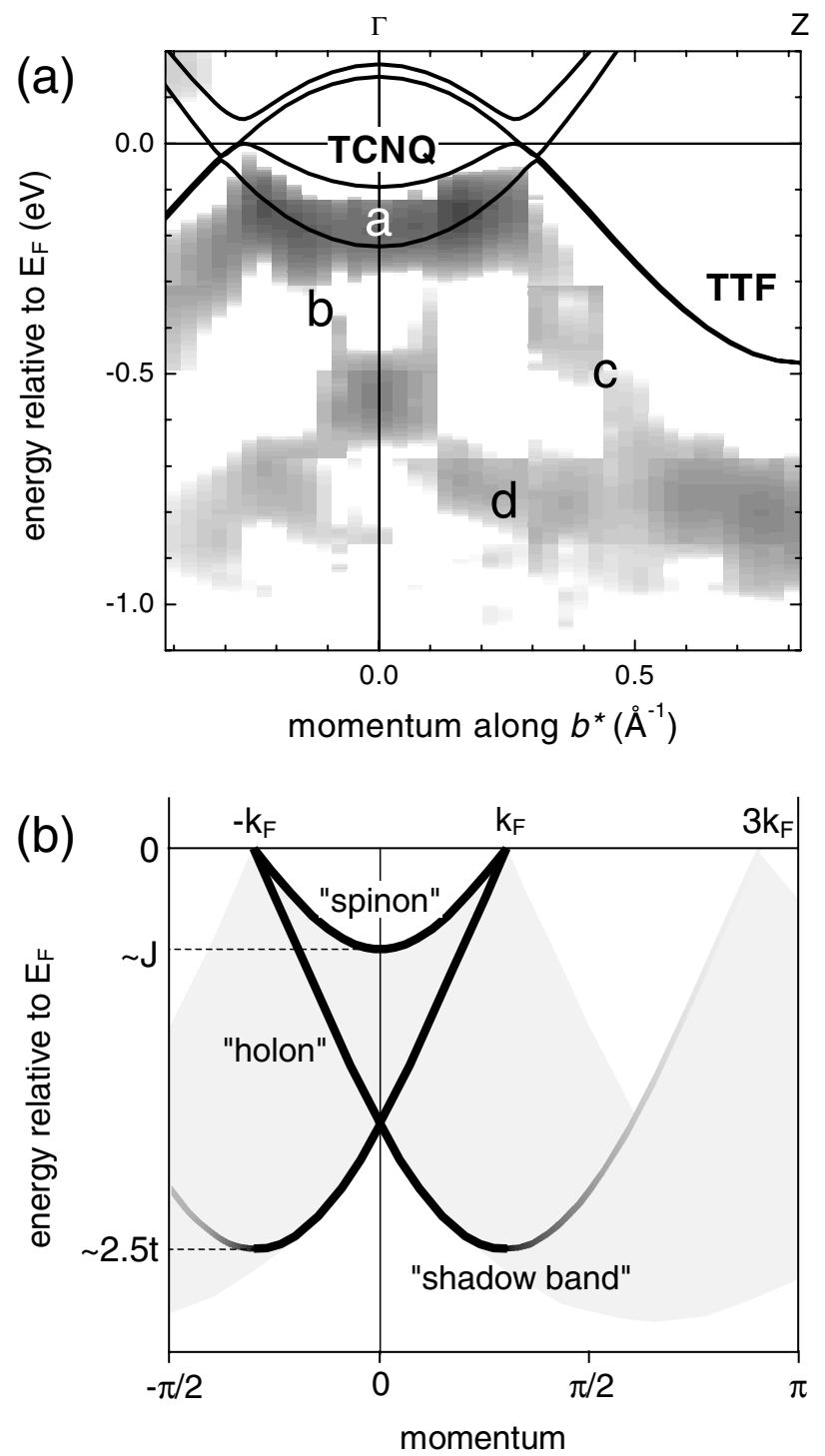

FIG. 3. (a) Gray-scale plot of the ARPES dispersions (see text for details). Also shown are the conduction bands obtained from density functional band theory [18]. (b) Schematic electron removal spectrum of the 1D Hubbard model at finite doping (band filling $n<2 / 3)$. conflict with the observed Fermi vector: The additional charge of a completely occupied surface state would severely affect the delicate charge balance between the TTF and TCNQ bands and shift the surface Fermi vector notably from its bulk value, which is not observed. An alternative explanation of $d$ as an umklapp image of the TTF band induced by long-ranged Peierls fluctuations [19] is ruled out due to the lack of other evidence of backfolding in the data.

In view of the strong correlation effects encountered in other properties of TTF-TCNQ we now compare the ARPES dispersions to the electron removal spectra of the 1D Hubbard model at finite doping, schematically depicted in Fig. 3(b). The (photo)hole generated by the removal of an electron decays into two collective excitations with separate dynamics, a spinon characterized by a spin quantum number and a spinless holon carrying the charge [1]. As a consequence the electron removal spectrum consists of a broad continuum determined by the phase space available for spinon-holon decomposition, indicated by the gray-shaded region in Fig. 3(b). However, dispersive singularities may appear [solid lines in Fig. 3(b)] for certain decompositions of the real hole. For example, the low binding energy singularity arises from hole fractionalization into a holon bound to the Fermi level and a propagating spinon; it therefore directly reflects the dispersion of a bare spinon, whose bandwidth scales with the exchange integral $J$. It is therefore referred to as the "spinon" branch. The "holon" branch corresponds to a spinon pinned to the Fermi level and a propagating holon. It crosses the Fermi level at $k_{F} \pm 2 k_{F}$ [3,7]. From its energy minimum at $k_{F}$ up to $3 k_{F}$ (referred to as "shadow band" in Ref. [3]) it decreases strongly in intensity until it becomes barely distinguishable from the diffuse background. Initially this spectral behavior was derived for the strong coupling limit $(U \gg 4 t)$ of the 1D Hubbard model [3,20]. Very recently it has been shown that it also holds for moderate interaction strengths of $U \sim 4 t$ as relevant for TTF-TCNQ [6,7].

Comparing Figs. 3(a) and 3(b), one finds a surprisingly good agreement of the TCNQ-related ARPES dispersions with the Hubbard model spectrum. The experimental structures $a$ and $b$ closely resemble those of the theoretical spin and charge branches, respectively [21]. Furthermore, the as yet unidentified structure $d$ can be well accounted for as the shadow band of the Hubbard model, at least for not too large $k$ vectors. Its upwards dispersion beyond $k_{F}$ and its eventual $3 k_{F}$ crossing are not observed, probably due to the theoretically predicted loss of weight at larger $k$ and the interfering TTF band. Within the Hubbard model interpretation the experimental dispersions thus give direct spectroscopic evidence of spin-charge separation in TTF-TCNQ.

The Hubbard model interpretation of the experimental dispersions is further supported by the temperature dependence of the ARPES spectra measured at $k_{F}$, shown in Fig. 4. With increasing temperature from 60 to $260 \mathrm{~K}$ the spectra reveal a dramatic shift of intensity from the 


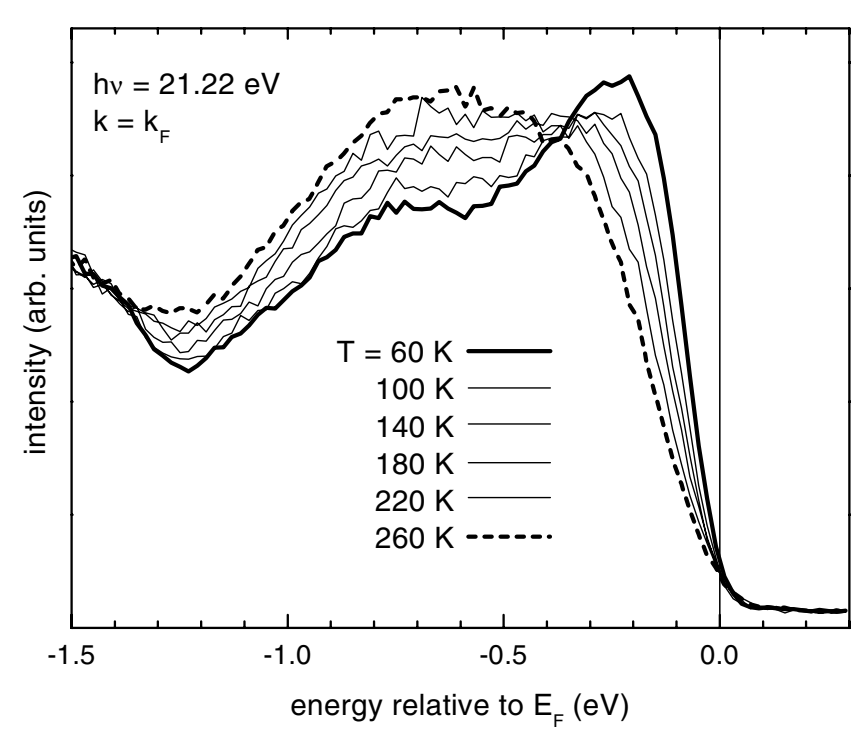

FIG. 4. Temperature dependence of the spectrum at $k=k_{F}$. Note the dramatic transfer of weight from low to high binding energy with increasing temperature.

low binding energy peak $(a / b)$ to structure $d$ at $0.75 \mathrm{eV}$. The effect is fully reversible and conserves the total spectral weight within experimental accuracy. The strong temperature dependence was already reported in Ref. [16], but the weight conservation had been overlooked there due to a different intensity normalization [22]. A weight transfer over an energy scale so much larger than $k_{B} T$ cannot be accounted for by conventional electron-phonon coupling or the effect of Peierls fluctuations. On the other hand, calculations of the temperature-dependent spectrum of the $t J$ model at quarter filling predict a redistribution of spectral weight on a scale of $t$ already for temperatures $k_{B} T \ll t$ [4], exactly as observed in our data. A similar temperature-dependent weight transfer has recently been reported for ARPES spectra of the 1D Mott-Hubbard insulator $\mathrm{Na}_{0.96} \mathrm{~V}_{2} \mathrm{O}_{5}$ [23] but without a resolution of individual spinon and holon peaks.

In summary, the unusual behavior of the ARPES spectra of TTF-TCNQ is consistent with the 1D Hubbard model at finite doping, thus reflecting spectral signatures of spin-charge separation over an energy scale of the conduction bandwidth. We note, however, that the nearly linear spectral onset at low energies is not reproduced by the simple Hubbard model. Close to $E_{F}$ the density of states of an interacting 1D metal is expected to follow a $\left|E-E_{F}\right|^{\alpha}$ power law behavior. With only on-site interactions the Hubbard model yields $\alpha \leq 1 / 8$ [1], at variance with the ARPES data. Exponents of up to $\alpha \sim 1$ become possible for an extended Hubbard model including nearest- and next-nearest-neighbor interactions [24]. Based on the strong $2 k_{F}$ correlations in TTF-TCNQ also the Luther-Emery (LE) model has been suggested to provide an adequate description of its low-energy spectrum [25]. Whether the extended Hubbard model or the LE model is compatible with the observed highenergy behavior is presently unknown and calls for further theoretical work.

We thank S. Hao, Th. Finteis, C. Janowitz, and G. Reichardt for technical support at BESSY, and J. M.P. Carmelo, A. Kampf, A. Muramatsu, K. Penc, and J. Voit for stimulating discussions. This work was supported by the DFG (CL 124/3-1 and SFB 484) and the BMBF (Grant No. 05SB8TSA2).

[1] J. Voit, Rep. Prog. Phys. 58, 977 (1995).

[2] V. Meden and K. Schönhammer, Phys. Rev. B 46, 15753 (1992).

[3] K. Penc, K. Hallberg, F. Mila, and H. Shiba, Phys. Rev. Lett. 77, 1390 (1996).

[4] K. Penc, K. Hallberg, F. Mila, and H. Shiba, Phys. Rev. B 55, 15475 (1997).

[5] J. M. P. Carmelo, N. M. R. Peres, and P. D. Sacramento, Phys. Rev. Lett. 84, 4673 (2000).

[6] D. Sénéchal, D. Perez and M. Pioro-Ladrière, Phys. Rev. Lett. 84, 522 (2000).

[7] J. M. P. Carmelo and J. M. B. Lopos dos Santos, L. M. Martelo and P. D. Sacramento (to be published).

[8] M. Grioni and J. Voit in Electron Spectroscopies Applied to Low-Dimensional Materials, edited by H. Starnberg and H. Hughes (Kluwer, Dordrecht, 2000), Vol. 1.

[9] G.-H. Gweon et al., J. Electron Spectrosc. Relat. Phenom. 117-118, 481 (2001).

[10] T. J. Kistenmacher, T. E. Phillips, and D. O. Cowan, Acta Crystallogr. Sect. B 30, 763 (1974).

[11] S. Kagoshima, H. Nagasawa, and T. Sambongi, OneDimensional Conductors (Springer, Berlin, 1987), and references therein.

[12] J. B. Torrance, Y. Tomkiewicz, and B. D. Silverman, Phys. Rev. B 15, 4738 (1977).

[13] S. Hüfner, Photoemission Spectroscopy (Springer, Berlin, 1995).

[14] C. Janowitz et al., J. Electron Spectrosc. Relat. Phenom. 105, 43 (1999).

[15] R. Claessen et al., Physica B (to be published).

[16] F. Zwick et al., Phys. Rev. Lett. 81, 2974 (1998).

[17] Our $Z$ point is identical to the $Y$ point of Ref. [16].

[18] Obtained with the WIEN97 code (Vienna University of Technology, 1997). [Improved version of P. Blaha, K. Schwarz, P. Sorantin, and S. B. Trickey, Comput. Phys. Commun. 59, 339 (1990)].

[19] J. Schäfer et al., Phys. Rev. Lett. 87, 196403 (2001).

[20] J. Favand et al., Phys. Rev. B 55, R4859 (1997).

[21] In this interpretation the doublet character of the TCNQ bands predicted by band theory is assumed to be unresolved in the ARPES spectra.

[22] Our spectra are normalized to incoming photon flux, which incidentally leads to an alignment of the spectra for binding energies $\gtrsim 1.3 \mathrm{eV}$, independent of temperature.

[23] K. Kobayashi et al., Phys. Rev. Lett. 82, 803 (1999).

[24] A. K. Zhuravlev and M. I. Katsnelson, Phys. Rev. B 64, 033102 (2001).

[25] J. Voit, Eur. Phys. J. B 5, 505 (1998). 